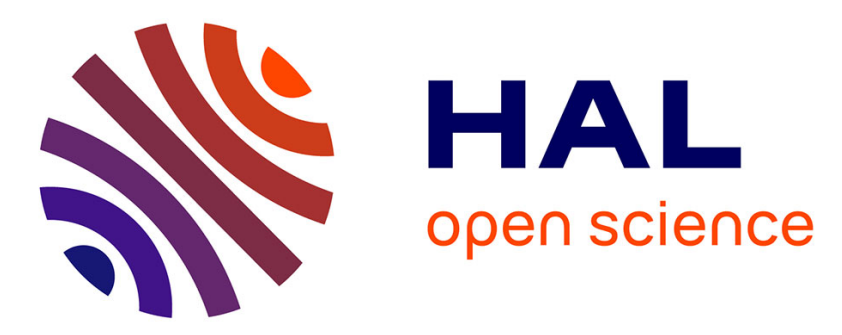

\title{
3-D characterization of weathered building limestones by high resolution synchrotron X-ray microtomography
}

Olivier Rozenbaum

\section{To cite this version:}

Olivier Rozenbaum. 3-D characterization of weathered building limestones by high resolution synchrotron X-ray microtomography. Science of the Total Environment, 2011, 409, pp.1959-1966. 10.1016/j.scitotenv.2011.02.014 . insu-00582895

\section{HAL Id: insu-00582895 https://hal-insu.archives-ouvertes.fr/insu-00582895}

Submitted on 4 Apr 2011

HAL is a multi-disciplinary open access archive for the deposit and dissemination of scientific research documents, whether they are published or not. The documents may come from teaching and research institutions in France or abroad, or from public or private research centers.
L'archive ouverte pluridisciplinaire HAL, est destinée au dépôt et à la diffusion de documents scientifiques de niveau recherche, publiés ou non, émanant des établissements d'enseignement et de recherche français ou étrangers, des laboratoires publics ou privés. 
3-D characterization of weathered building limestones by high resolution synchrotron X-ray

$$
\text { microtomography }
$$

O. Rozenbaum

\author{
Olivier. ROZENBAUM \\ Assistant professor, \\ Institut des Sciences de la Terre d'Orléans (ISTO) \\ UMR6113 CNRS/Université d'Orléans \\ $1 \mathrm{~A}$, rue de la Férollerie \\ 45071 Orléans Cedex 2 \\ France \\ rozenbaum@cnrs-orleans.fr \\ Phone: 332-38-25-52-44 \\ Fax: 332-38-63-64-88
}




\begin{abstract}
Understanding the weathering processes of building stones and more generally of their transfer properties requires detailed knowledge of the porosity characteristics. This study aims at analyzing three-dimensional images obtained by X-ray microtomography of building stones. In order to validate these new results a weathered limestone previously characterised (Rozenbaum et al., 2007) by two-dimensional image analysis was selected. The 3-D images were analysed by a set of mathematical tools that enable the description of the pore and solid phase distribution. Results show that 3-D image analysis is a powerful technique to characterise the morphological, structural and topological differences due to weathering. The paper also discusses criteria for mathematically determining whether a stone is weathered or not.
\end{abstract}

\title{
Key word
}

Stone weathering, 3-D image analysis, X-ray microtomography, porous media, segmentation, topology. 


\section{Research aims}

Weathering of building stones is a widespread phenomenon observed throughout the world. Every stone extracted from a quarry is subjected to deterioration due to the action of external environmental agents (physical, chemical and biological) such as rain, pollution, sunlight, or freezing/thawing cycles. Furthermore, every stone has its own morphological, petrophysical, structural and topological characteristics and reacts differently, depending on its environment: two identical stones placed in two distinct environments can develop two different weathering facies. A prerequisite for modeling weathering mechanisms, therefore, is to characterize weathered stones from buildings and unweathered stones extracted from quarries or from buildings, but in places where the sampled stones can be assumed to be unweathered by environmental agents. As water transfer is one of the main factors involved in stone weathering and is directly linked to the porous network, the objective here is to study the morphological, structural and topological characterization of stones. To achieve this, a promising method is 3-D image analysis using high resolution X-ray microtomography.

\section{Introduction}

In a previous article (Rozenbaum et al., 2007), an experimental approach to characterize weathered stones by using some classical techniques was reported, and certain image analysis tools were presented. Image analysis provides a huge quantity of data, offering important insights to characterize the porous medium. Within recent decades, 2-D image analysis (images generally acquired by SEM) has been commonly used to characterize different porous materials such as porous silica, soils, concrete and stones (e.g. Cousin et al., 1996; D’acqui et al., 1992; Levitz and Tchoubar, 1992; Török and Rozgonyi, 2004). However, the transfer properties of porous media are related to 3-D characteristics of the porous network which cannot be appropriately described by 2-D analysis since the latter 
cannot provide complete topological information on the porous network. Since the middle of the '90s, 3-D X-ray microtomography has significantly improved and has been widely used in many fields of investigation such as materials science and geology (Bentz et al., 2000; Biswal et al., 1999; Burlion et al., 2006; Cnudde and Jacobs, 2004a; Coles et al., 1998; Ketcham and Carlson, 2001; Lindquist, 1999; Lindquist, 2002; Pierret et al., 2002; Spanne et al., 1994). Sandstones, for example, have been extensively characterized and the subject of many publications (Biswal et al., 1999; Coles et al., 1998; Spanne et al., 1994). This technique is underused, however, in the field of building conservation, despite the fact that it can provide this branch of research with new information, as has been shown recently (Cnudde et al., 2004b; De Graef et al. 2005). In these two studies, X-ray computed tomography (CT) analysis was used to visualize bacterial weathering of concretes and stones or to locate water repellents and consolidants in building stones. The technique was applied with a resolution of about 10 $\mu \mathrm{m}$, as this resolution was considered appropriate for building stones. However, part of the porous network was not analyzed because the corresponding pores were much smaller than the resolution used. For some building stones such as the one selected for this study, a high proportion of the porous network requires a higher resolution than $10 \mu \mathrm{m}$ if it is to be analyzed properly (i.e. less than $1 \mu \mathrm{m}$ voxel size) and a high-contrast image. Currently, these specifications require the use of synchrotron radiation (Gallucci et al., 2007, Maire et al., 2007; Parra Denis et al., 2008; Rolland du Roscoat et al., 2007; Tafforeau et al. 2007) coupled with reliable image analysis.

The present work focuses on the analysis of 3-D images using mathematical tools and illustrates the interest of this kind of analysis in building stone conservation. The stone selected was the Tuffeau limestone which had been studied earlier (e.g. Rozenbaum et al., 2007 and references herein) by classical techniques. 


\section{Material and methods}

\subsection{Materials}

Tuffeau limestone is a yellowish-white porous sedimentary limestone, mainly composed of calcite, silica in the form of opal cristobalite-tridymite and quartz, and some secondary minerals such as clays and micas. The unweathered Tuffeau stone from a quarry (Saint-Cyren-Bourg, France) studied in this paper was mainly composed of calcite $\left(0.503 \mathrm{~g} \mathrm{~g}^{-1}\right)$, and silica $\left(0.452 \mathrm{~g} \mathrm{~g}^{-1}\right)$ and the total porosity was 0.481 . It was a multi-scale porous medium since the equivalent pore size distribution ranged from 0.01 to $50 \mu \mathrm{m}$ (Rozenbaum et al., 2007).

The weathered Tuffeau stone selected came from the Saint-Donatien church located in Orléans (France). Note that the origin of weathering was undetermined and that no building or environmental monitoring was done before sampling. Blocks were extracted from the northeast wall of the bell tower at an elevation of $20 \mathrm{~m}$. Sampling occurred during restoration of the church, which consisted in replacing entire blocks by new ones. The exposed surface was a grey crust harder than the stone core, and the first $3 \mathrm{~cm}$ below the surface were crumbly with micro-cracks parallel to the surface. Gypsum was present in the first $30 \mathrm{~mm}$ below the surface but mainly concentrated in the first $20 \mu \mathrm{m}$, corresponding to the grey crust (Rozenbaum et al., 2007). A morphologically representative block was selected and two sets of samples were then constituted. The first set was taken from the 0-3 mm layer below the exposed surface and corresponds to a weathered Tuffeau stone. It consisted of three samples W0-1, W1-2 and W2-3 from the 0-1, 1-2 and 2-3 mm layers below the exposed surface (Fig. 1). The second set was taken from the $80-81 \mathrm{~mm}$ layer underneath the exposed surface (UW sample) and was considered as a sample of unweathered Tuffeau stone based on the criteria established in a previous study (Rozenbaum et al., 2007), where "unweathered" was considered to mean that part of the stone (in depth) had undergone very little (or no) evolution 
and was in almost the same state as at the building of the wall. This was the case far from the surface (80-81 $\mathrm{mm}$ in the present study, following the previous article) where the stone had rarely (or never) undergone wetting/drying cycles. Unweathered stones were therefore considered as reference stones.

\subsection{Obtaining the 3-D images}

X-ray microtomography is a technique which enables high-resolution evaluation of 3D structural information. The microtomography images were collected at the MS-X04SA-Tomo beam line of SLS (Swiss Line Source, Stampanoni et al., 2002) and the energy used was 12 $\mathrm{keV}$. Successive rotations of the sample, 1500 projections, corresponding to 1500 angular positions ranging between $0^{\circ}$ and $180^{\circ}$, were acquired by a camera with $1024 \times 1024$ pixels. The good quality of the beam made it possible to image samples at a $0.7 \mu \mathrm{m}$ pixel resolution. In order to remain within the field of view of the detector and to avoid additional artifacts, the samples had to measure less than $700 \mu \mathrm{m}$. The samples were prepared as cylindrical cores, $700 \mu \mathrm{m}$ in diameter, and were mounted on a vertical rotator on a goniometric cradle. Preparing limestone samples with these dimensions requires particular care. The samples were resin impregnated in order to make a $700 \mu \mathrm{m}$ thin section (Bruand and Prost, 1987). The thin section was then cut into $700 \mu \mathrm{m}$ matches with a diamond saw, and the edges of the matches were filed down to obtain quasi cylinders.

Imaging time was approximately 120 minutes. A filtered back-projection algorithm was used to reconstruct horizontal slices $0.7 \mu \mathrm{m}$ thick from the projections. Stacking the 1024 slices reconstructed produced a 3-D volume (Fig. 2). Each pixel from a tomographic slice corresponds to a specific voxel in the 3-D image. The whole data set corresponding to one 3D image has an approximate size of one Gbyte (1024 $4^{3}$ voxels). Each voxel was characterized 
by its grey level (ranging from 0 to 255 for an 8-byte image) which depended on the X-ray attenuation coefficient of the element (Baruchel et al. 2000).

\subsection{Image analysis and segmentation}

Image quantification involves correctly segmenting the solid and porous phases. However, it was not possible to obtain a direct threshold of the raw images from their histogram (Fig. 3, dot curve), as the expected three phases (pore, silica, and calcite) could not be identified. In an ideal image without any noise, two grey levels (1l and lh) would be selected. Hence, all the voxels belonging (i) to the $[0,11]$ range would represent the pore phase, (ii) to the $[11,1 \mathrm{~h}]$ range would represent the silica phase and (iii) to the [1h, 255] range would represent the calcite phase. In the present case of a real image, the presence of noise (Le Trong et al., 2008) meant that these two grey levels, which distinguish each phase from the other two, could not be selected. Unfortunately, the algorithms generally used for 2-D image segmentation were not efficient for most of the 3-D images due to finite resolution effects of noise, leading to similar grey levels for different phases. Many authors used their own algorithm, depending on the type of materials studied (e.g. Le Trong et al., 2008; Lindquist, 2002; Vignoles, 2001). Initial studies of our complex 3-D images showed that these algorithms were inefficient and new procedures to binarize the images were therefore created (Le Trong et al., 2008).

Briefly, a two-step filtering approach was chosen. Alternate sequential filters were first used to remove the noise but, as they destroy the structural components smaller than the structuring element used, they cannot be pursued far enough. As a second step in the filtering process, a mosaic operator, based on a pragmatic but efficient marker determination, was therefore implemented to simplify the images further. This yielded the mosaic (Fig. 4b) of flat zones, which was much simpler than the starting image (Fig. 4a) and straightforward to segment. 
Using the histogram (Fig. 3, solid curve) the threshold levels were easily identified (as the minimum values between the peaks). The values of 11 and $1 \mathrm{~h}$ for each sample are reported in table 1.

\subsection{Estimators}

Different estimators were then calculated on the segmented images. Most of these estimators were described in (Rozenbaum et al., 2007) for 2-D images but their extension to 3-D images was straightforward. The estimators are briefly explained below; the reader is referred to references (Levitz and Tchoubar, 1992) and (Rozenbaum et al., 2007) for more detail.

\section{Porosity}

As the thresholds were distinguished, the porosity, calcite and silica volume fraction could be calculated (Table 1). The fraction of phase i within the volume was defined as: $\phi_{i}=\frac{V_{i}}{V_{T}}$

where $V_{i}$ and $V_{T}$ are the volume of phase $i$ and the total volume of the sample, respectively. For a 3-D digitized medium, $V_{i}$ and $V_{T}$ are the number of voxels corresponding to phase $i$ and to all the phases, respectively. Hence, total porosity $\phi$ is calculated if phase $i$ is the pore phase.

\section{Chord distributions}

Chord distributions are stereological tools used to describe the interface between pore and solid phases (Levitz and Tchoubar, 1992; Rozenbaum et al., 2007). The chord distribution gives the probability of having a chord length between $r$ and $r+d r$. Chord distribution can be calculated either for the pores $\left(f_{p}(r)\right.$, or pore chord distribution) or for the solid $\left(f_{s}(r)\right.$, or solid chord distribution). Furthermore, the first momentum of $f_{p}(r)$ (called $1_{p}$ ) and the first momentum of $\mathrm{f}_{\mathrm{s}}(\mathrm{r})\left(\right.$ called $\left.\mathrm{l}_{\mathrm{s}}\right)$ are defined as: 
$1_{p}(r)=\int r f_{p}(r) d r$

and,

$1_{s}(r)=\int r f_{s}(r) d r$.

The values of $1_{\mathrm{p}}$ and $1_{\mathrm{s}}$ can be considered to be the estimators of the mean size of the pore and solid phases.

Due to digitizing, the smallest pores (or solids) were badly defined and did not correspond to real objects: as the chord distributions for $\mathrm{r}<3$ pixels presented a linear increase, they were therefore not taken into account (Cousin et al., 1996; Rozenbaum et al., 2007).

\section{Autocorrelation function}

The autocorrelation function $R_{Z}(\vec{r})$ is a statistical description of a porous medium (Levitz and Tchoubar, 1992; Rozenbaum et al., 2007). This function can be interpreted as the probability of finding two randomly selected points that are both in the same phase. For an isotropic medium with $r=|\vec{r}|, R_{Z}(\vec{r})=R_{Z}(r)$, the autocorrelation function depends on distance alone. Otherwise, the one dimensional autocorrelation functions for $\overrightarrow{\mathrm{r}}$ parallel to the $\mathrm{Ox}$, Oy or $\mathrm{Oz}$ axis (where $\mathrm{Ox}, \mathrm{Oy}, \mathrm{Oz}$ define an orthogonal reference), respectively noted $\mathrm{R}_{\mathrm{Zx}}, \mathrm{R}_{\mathrm{zy}}$ and $R_{\mathrm{Zz}}$, give information about isotropy or anisotropy, since pore space anisotropy is revealed as a disparity between the one-dimensional autocorrelation functions along different directions (Frykman et Rogon, 1993). Another characteristic length scale is the correlation length L, defined as the integral of the autocorrelation function. It represents a length scale characteristic of the spatial structure (Ioannidis et al., 1996):

$$
L=\int_{0}^{\infty} R_{Z}(r) d r
$$


For anisotropic materials, $\mathrm{L}_{\mathrm{x}}, \mathrm{L}_{\mathrm{y}}$ and $\mathrm{L}_{\mathrm{z}}$ are defined accordingly from $\mathrm{R}_{\mathrm{Zx}}, \mathrm{R}_{\mathrm{zy}}$ and $R_{\mathrm{Zz}}$.

\section{Topological analysis}

In addition to these estimators, which are also valid in 2-D, the topology of the pore space is a fundamental property as regards flow and transport in porous media (and can only be estimated on a 3-D volume), since pore connectivity strongly influences these properties. Fortunately the main advantage of X-ray microtomography resides in 3-D structure analysis and topology analysis. The Hoshen-Kopelman algorithm (6-connectivity) was used to identify (or label) each pore with a number (Hoshen and Kopelman, 1976). This is also a simple and efficient cluster identification algorithm for identifying connected clusters on a lattice (percolation theory). The size is determined by the number of voxels in each cluster. This algorithm was also used to label each calcite cluster and each silica cluster.

The basic topological properties are the number $\mathrm{N}$ of isolated pores, the number of redundant connections $\mathrm{C}$ within the pore space, and the number of completely enclosed cavities $\mathrm{H}$ (Vogel and Roth, 2001). In other words, C represents the number of bonds that can be removed without creating an additional isolated cluster. The Euler-Poincaré characteristic $\varepsilon_{\mathrm{V}}$ was then computed as $\varepsilon_{\mathrm{V}}=\mathrm{N}-\mathrm{C}+\mathrm{H}$. For the topology of porous media such as stones, the quantity $\mathrm{H}$ is of minor importance since solid grains or particles completely surrounded by pores are scarce. H was therefore set to zero within the previous equation (Vogel and Roth, 2001). As this characteristic is related to the total volume (V, in voxel units) and in order to compare images of different sizes, the specific Euler-Poincaré number was defined as $\chi_{\mathrm{v}}=\varepsilon_{\mathrm{v}} /$ $\mathrm{V}$. This number can be considered as a connectivity measure: the greater the number is, the less connectivity can be expected. 


\section{Analysis and Discussion}

\subsection{Pore and solid phase morphology}

As the grey level value of a voxel was linked to the X-ray absorption of the sample at the voxel position (Baruchel et al., 2000), the pores appeared in dark grey, the silica compounds in medium grey and the calcite compounds in light grey (Fig. 2). The different phases were easily distinguishable to the naked eye: calcite was present in the form of large irregular grains (sparitic calcite) or small grains that look like crumble (micritic calcite), whereas silica had the form of large quartz crystals or small spheres of opal. The fine structure of the latter was not visible at this pixel size, but their global shape made it possible to distinguish their spatial distribution. For example, on the upper right corner of Fig. 2f, some opals embedded in a large calcite grain can be seen.

Simple observation of the 3-D images such as those presented on Fig. 2 showed that the W0-1 sample was strongly altered due to the presence of numerous large cracks. For the W1-2 and W2-3 samples, some cracks or large pores were visible but, at first glance, the weathering was far less severe than for W0-1. Some pores were visible on the UW sample, as expected for an unweathered Tuffeau stone (no cracks or large pores). The four samples could not be distinguished by their solid compounds morphologies, only by the presence or absence of cracks or pores i.e. the UW sample was compact, the W1-2 sample slightly less compact (due to the presence of some large pores), the W2-3 sample was a little less compact again, and the W0-1 sample was not compact at all.

\subsection{Porous phase analysis.}

Average porosities calculated for the four 3D images are reported in Table 1 (volume fraction of pores). The porosity determined by image analysis for the unweathered stone $(0.228)$ was smaller than the porosity determined from the density measurement conducted in 
(Rozenbaum et al., 2007), where a porosity of 0.481 was recorded for this sample. This difference may be related to the small pores which were not taken into account in the 3D image analysis because they were smaller than the pixel size $(0.7 \mu \mathrm{m})$. These small pores correspond partly to those resulting from the assemblage of the silica and calcite grains, the structure and morphology of these grains (e.g opal spherolites) as well as fine particles such as clays. Nevertheless, a comparative study of these 3D stone images enabled weathering features to be detected.

First of all, as shown in Fig. 5, the pore and solid chord distributions for all samples presented exponential decreases. This type of porous medium, in which both the porous and solid chord distributions are exponential, is called a long-range random medium (Levitz and Tchoubar, 1992; Rozenbaum et al., 2007). In other words, the pore and solid phases in the 3D image were randomly distributed. The absence of correlation peaks demonstrated that the pore and solid phases were heterogeneously distributed in size.

As shown on Table 1, the porosity of the surface sample (W0-1) was high (0.467) in comparison with the other porosities. In particular, from the exposed surface, porosities were much lower in the 1-2 mm layer (0.289) and in the 2-3 mm layer (0.296) (that were both very close) than in the $0-1 \mathrm{~mm}$ layer $(0.467)$. The porosity of the unweathered Tuffeau stone (i.e. between 80 and $81 \mathrm{~mm}$ below the exposed surface) was lower than that of the weathered Tuffeau stones ( 0.228 compared to the recorded porosity in the $0-1,1-2$ and $2-3 \mathrm{~mm}$ layers). Similarly, the pore chord distribution (Fig. 5a) of the surface sample (0-1 mm layer) extended to the longest chord range (about $310 \mu \mathrm{m}$ for a probability of $2 \times 10^{-6}$ ) while the pore chord distributions of the 1-2 $\mathrm{mm}$ and 2-3 mm layer samples extended to about $200 \mu \mathrm{m}$ for a probability of $2 \times 10^{-6}$. Finally, the pore chord distribution of the $80-81 \mathrm{~mm}$ layer extended to 
the shortest chord range (about $130 \mu \mathrm{m}$ for a probability of $2 \times 10^{-6}$ ). This behaviour was in accordance with that of the total porosities and suggests that, statistically, there were strong modifications in the pore distribution within the W0-1 sample and non-negligible porous modifications in the W1-2 and W2-3 samples. Using the classification used in (Rozenbaum et al., 2007) and the results presented above and in the following (visual analysis of 3D volumes, porosity, chord length distributions, anisotropy etc...), the W0-1 sample could be considered as strongly weathered and the W1-2 and W2-3 samples could be considered as moderately weathered.

Furthermore, the Hoshen-Kopelmann algorithm showed that for each sample one percolating pore cluster existed and accounted for the quasi-totality (about 99\%) of the total pore volume, demonstrating that the porous media can be considered as fully connected. This was confirmed by the negative values of the specific Euler-Poincaré characteristics $\chi_{\mathrm{V}}$ (Table 1). The percolating pore cluster (Table 1$)$ was the highest in the $0-1 \mathrm{~mm}$ layer $\left(\approx 2.5 \times 10^{8}\right.$ voxels) and was the lowest in the $80-81 \mathrm{~mm}$ layer, i.e. in the unweathered Tuffeau stone $\left(\approx 1.2 \times 10^{8}\right.$ voxels). The remaining unconnected pores ( $4 \%$ to $0.4 \%$ of the total pore volume, depending on the sample) consisted of small clusters with sizes ranging from a few voxels up to about $80 \times 10^{3}$ voxels (i.e. considerably smaller than the percolating pore volume). The quantity of these remaining unconnected pores was the lowest for the weathered sample W0-1 $(\approx 3400)$ and was the highest for the unweathered UW sample ( $\approx 6200)$. In fact, because of weathering, initially unconnected pores were connected together, resulting in larger pore clusters. This also led to a decrease in the number of unconnected pores and an increase in the main percolating pore cluster volume.

\subsection{Isotropy/anisotropy}


The autocorrelation functions $\left(\mathrm{R}_{\mathrm{Zx}}, \mathrm{R}_{\mathrm{Zy}}\right.$ and $\left.R_{\mathrm{Zz}}\right)$ and the average value $R_{\mathrm{Z}}$ for the UW sample are presented in Fig. 6a. The Oxy plane was taken parallel to the stone surface, the axis $\mathrm{Oz}$ being perpendicular to the rod sample (Fig. 1). All these functions presented a decreasing behaviour without any particular correlation. For the UW sample, no particular disparity was observed for the one-dimensional autocorrelation functions along the orthogonal x-, y-, z- directions and $R_{z}$ (Fig. 6a) and between the correlation lengths (Table 2). This means that the UW sample presents no, or only very slight, anisotropies. In contrast, the W0-1 sample autocorrelation functions (Fig. 6b) and the correlation lengths (Table 2) presented a non-negligible anisotropy. As shown on Fig. 2, this anisotropy was caused by cracks. The anisotropy decreased for the W1-2 sample and decreased yet further for W2-3 (curves not shown here), becoming nil for the UW core sample, as described previously.

\subsection{Solid phase analysis}

Calcite volume fractions (Table 1) were quasi constant (about 0.29) whatever the sample, except for the W0-1 sample which had a much lower volume fraction (0.19). In other words, the calcite volume fractions of the moderately weathered samples (W1-2 and W2-3) were similar but, surprisingly, were also similar to the calcite volume fraction of the unweathered stone. The silica volume fractions were the highest for the unweathered sample $(0.48)$ and decreased for the moderately weathered stones (about 0.42 ). Finally the silica volume fraction of the W0-1 sample was the smallest (0.35).

The solid chord distribution (Fig. 5b) in the $0-1 \mathrm{~mm}$ layer extended to the shortest chord range (about $260 \mu \mathrm{m}$ for a probability of $2.10^{-6}$ ), whereas the solid chord distributions of the W1-2 and UW samples were both similar and extended to the highest chord length (about $425 \mu \mathrm{m}$ for a probability of $\left.2 \times 10^{-6}\right)$. Moreover, the solid chord distribution of the W2-3 sample 
extended to an intermediate chord length (about $375 \mu \mathrm{m}$ for a probability of $2 \times 10^{-6}$ ). This result meant that the distances between two interfaces within the solid phase were statistically equivalent in the W1-2 and UW samples and were both larger than (i) the W2-3 sample above $250 \mu \mathrm{m}$ and (ii) the W0-1 sample above $75 \mu \mathrm{m}$.

The Hoshen-Kopelmann algorithm showed that for each sample, one percolating calcite cluster and one silica cluster existed. In other words, the silica grains (resp. calcite) adhered to each other in order to build a cohesive backbone that guarantees, a priori, the integrity of the stone. As for the calcite volume fraction analysis, the volumes of the W1-2, W2-3 and UW calcite clusters were quasi constant, about $1.5 \times 10^{8}$ voxels, accounting for more than $99 \%$ of the total calcite fraction (Table 1). On the other hand, the W0-1 sample had a much lower calcite cluster (about $0.4 \times 10^{8}$ voxels) representing "only" $37 \%$ of the total calcite fraction. The unconnected grain parts were similar $(0.4 \%)$ for the W1-2, W2-3 and UW samples, and some large unconnected large calcite grains (about $2 \times 10^{6}$ to $5 \times 10^{5}$ voxels i.e. much less than the percolating calcite volume) were found. Although the number of calcite clusters was very similar (about 2900) whatever the sample, the unconnected grains in the W0-1 sample were 10 times larger than in the other samples. This may result from the mechanical constraints suffered and/or partial dissolution of the initial calcite cohesive backbone of the unweathered Tuffeau stone as it appears in the $80-81 \mathrm{~mm}$ stone, partially cracking this backbone into relatively large unconnected calcite grains.

The percolating silica cluster (Table 1$)$ was the smallest in the $0-1 \mathrm{~mm}$ layer $\left(\approx 1.9 \times 10^{8}\right.$ voxels $)$ and was the largest in the $80-81 \mathrm{~mm}$ layer $\left(\approx 2.6 \times 10^{8}\right.$ voxels $)$. The percolating silica clusters represent more than $99 \%$ of the total silica fraction for all the samples. The remaining unconnected silica grains consisted of about 4000 small grains with sizes ranging from a few 
voxels up to about $50 \times 10^{3}$ voxels for all the samples (i.e. much smaller than the percolating silica volume).

At this stage of analysis it seemed that some silica grains had been removed from their settings during the stone history. This phenomenon occurred, however, without any calcite dissolution for the moderately weathered stones.

\subsection{D/3D comparisons and weathering criteria}

It was possible to make some qualitative comparisons between the 3-D image data and the earlier 2-D image data (Rozenbaum et al., 2007), bearing in mind however that (i) the pixel and voxel sizes were different (2.8 and $0.7 \mu \mathrm{m}$, respectively) and (ii) the studied stone thickness from the surface exposed to environmental agents was also different: for the 2-D images the samples were 1.2 to $1.4 \mathrm{~mm}$ thick, while for the 3-D images the rod samples were “only" $0.7 \mathrm{~mm}$ thick.

The present results (variation of porosity according to depth, anisotropy etc...) are consistent with those recorded earlier and enable a more detailed analysis of the differences resulting from weathering (topological analysis). Moreover, as expected for stereological tools, the general trends of the chord length distributions were similar to the 2-D chord length analysis.

One criterion (among others) to characterise the degree of weathering in (Rozenbaum et al., 2007) was obtained from the solid chord distribution analysis. The classification used in (Rozenbaum et al., 2007) established that the solid chord distribution of moderately weathered or unweathered samples tended to extend to the longest chord length, while the solid chord distribution of strongly weathered samples tended to extend to the shortest chord 
length (due mainly to cracks and large pores caused by partial grain dissolution and or mechanical constraints such as freezing/thawing cycles). This classification seemed to remain valid as the W1-2, W2-3 and UW solid chord distributions (Fig. 2) extended to longer chord lengths than those of the W0-1 sample. It was observed that the W2-3 solid chord distribution was modified above $230 \mu \mathrm{m}$. In other words, the solid chord distributions of the W1-2 and W2-3 samples show a slightly different solid phase distribution. This is rather surprising, as the volume fraction of pore, calcite and silica (Table 1) and the pore chord distributions of these two samples were identical.

One hypothesis is that during weathering, cracks and pores separated mineral grains, thus breaking the continuity of the solid. The result was a statistical decrease in the solid phase (silica+calcite) on the long solid chord range for the W2-3 sample (Fig 5b). In contrast (the case of the W1-2 sample), cracks "pushed" the solid phase, resulting in a higher concentration of the solid phase within some localised zones. Within these zones the solid phase was more compact (Fig. 2d) than within the W2-3 sample. Nevertheless, this analysis did not enable any conclusions to be drawn about the resistance of these layers, since although the grains were in contact as shown here, this gave no information about the bond strengths between these grains.

This hypothesis explains the difference between the solid chord distributions of the W1-2 and W2-3 samples and also explains why this behaviour was not observed in the previous study but was revealed now. In the present investigation (3-D), the probed sample thickness was 0.7 $\mathrm{mm}$ while the probed depth in the previous article (2-D) was twice as high. The latter depth was sufficiently large to contain both large cracks and compact zones (Fig 1e in (Rozenbaum et al., 2007)) in all the moderately weathered samples considered. The W1-2 sample studied 
here represented the same moderately weathered zone but, due to the smaller depth sampling, W1-2 sample was situated in a zone without large or long cracks. Note that in spite of this lack of long cracks, the porosity of W1-2 sample was higher than the porosity of the UW sample, due to weathering processes. The W1-2 sample contained some compact solid zones which created large solid chords, but also some zones with large pores which also created large pore chords. The same observations can be made for the W2-3 sample except that the solid phase was slightly more dispersed by the porosity. This could explain the small number of cohesive multilayer structures macroscopically observed in the first few millimetres below the surface.

In the previous paper (Rozenbaum et al., 2007), the ratio of the first momentum of chord length distributions was pointed out as a possible indicator of weathering. The lower the ratio was, the stronger the weathering. This is in accordance with the measurements presented here: $1_{\mathrm{s}} / 1_{\mathrm{p}}(\mathrm{W} 0-1)=1.1$ and $1_{\mathrm{s}} / 1_{\mathrm{p}}(\mathrm{UW})=3.1$ (Table 2$)$, confirming the hypothesis that the W1-2 and W2-3 samples were moderately weathered as $1_{\mathrm{s}} / 1_{\mathrm{p}}(\mathrm{W} 1-2)=2.2$ and $1_{\mathrm{s}} / 1_{\mathrm{p}}(\mathrm{W} 2-3)=2.1$.

Nevertheless, if these ratios are indeed weathering estimators, what is the threshold limit to determine whether a stone is strongly, moderately or not at all weathered?

This requires a statistical analysis on more images. Only new images on weathered and unweathered stones will provide an answer to this question. This will be the next step and the subject of forthcoming microtomography measurements on the synchrotron.

\section{Conclusion}

The results recorded in this study confirm and complete the previous work (Rozenbaum et al., 2007) and show that 3-D image analysis can quantify calcite, silica and pore modifications in stones. These modifications are related to the morphological differences caused by weathering 
on a building limestone: weathering created cracks on the exposed surface and increased the porosity within the first millimetres. Compared to the unweathered Tuffeau stone (which appeared to be isotropic), the weathered Tuffeau stones appeared to be rather anisotropic, which probably led to changes in water transfer properties. One of the main advantages of a 3-D image is that a topological analysis of the different phases present can be performed. Thus, it has been shown here that percolating pore clusters existed for weathered and unweathered samples, accounting for $99 \%$ of the total porosity. This topological analysis showed that weathering connected initially closed porosities, increasing the pore cluster volumes. This increase in porosity (notably by cracks) removed silica grains from their settings, induced below the surface some constraints on the solid phases, and locally compacted the grains. Hence, 3-D image analysis along the weathering depth of a building stone enhances our understanding of weathering mechanisms and, in the longer term, of transfer properties.

Finally weathering criteria have been proposed, but only a statistical analysis on more samples will be able to confirm their validity. This statistical analysis will also make it possible to determine the REV of each sample (moderately or strongly weathered and unweathered) and hence prove whether the samples are representative. Indeed, the main drawback of this technique and the use of high resolution is that the higher the resolution, the smaller the sample size. This will be the aim of the next proposal addressed to a synchrotron X-ray microtomography beam line.

\section{Acknowledgements}


The author would like to thank Marco Stampanoni and Amela Grosso (PSI, SLS, Villingen) for scientific support concerning the tomography experiments and Ary Bruand (ISTO) for his comments and suggestions on this paper. 


\section{Figure captions}

Fig. 1: Schematic representation of the origin of the four cylindrical samples studied in this article. Three came from the weathered surface (between 0 and $3 \mathrm{~mm}$ ) while the fourth came from the unweathered core (between 80 and $81 \mathrm{~mm}$ ).

Fig. 2: 3-D images of the weathered tuffeau originating from a church. The images were visualized by using VG-Studio software (http://www.volumegraphics.com). The rod dimensions are $(\mathrm{a}, \mathrm{c}, \mathrm{e}, \mathrm{g})$ : radius $=420$ voxels, height $=1024$ voxels. These rods were also axially cut (b, d, f, h). Fig. $2 \mathrm{a}$ and $2 \mathrm{~b}$ : W1-0 sample from 0 to $1 \mathrm{~mm}$ depth, Fig. $2 \mathrm{c}$ and $2 \mathrm{~d}$ : W1-2 sample from 1 to $2 \mathrm{~mm}$ depth, Fig. $2 \mathrm{e}$ and 2f: W2-3 sample from 2 to $3 \mathrm{~mm}$ depth, Fig. $2 \mathrm{~g}$ and $2 \mathrm{~h}$ : UW sample from the core ( $80 \mathrm{~mm}$ from the surface).

Fig. 3: Greyscale histograms recorded for the original image and after the mosaic operator of the W0-1 sample shown in Fig. 2a. The [0-115] grey levels correspond to the pore phase, the [116-154] grey levels to the silica phase, and the [155-255] grey levels to the calcite phase.

Fig. 4: a) 2-D zoom of a non segmented $3-D$ image $(720 \times 570$ pixels). b) Image (a) after application of the alternate sequential filters and the mosaic operator. c) thresholding of image (b) where the pore phase is in white (the others are in black). d) thresholding of image (b) where the silica phase is in white (the others are in black). e) thresholding of image (b) where the calcite phase is in white (the others are in black).

Fig. 5: Comparison between a) the pore chord and b) the solid chord distributions.

Fig. 6: Autocorrelation functions of a) the UW sample and b) the W0-1 sample. 


\section{Table captions}

Table 1: Grey level threshold, volume fraction, volume of percolating cluster (voxels) and specific Euler number for the four samples studied. The latter was calculated by using the library QuantIm (Vogel, 2008).

Table 2: Ratios of chord length momentum, correlation length and anisotropy for the four samples studied. 


\section{References}

Baruchel J., Buffiere J.Y., Maire E., MerleP., Peix G., X-Ray tomomography in material science, Paris, Hermes, 2000.

Bentz D.P., Quenard D.A., Kunzel H.M., Baruchel J., Peyrrin F., Martys N.S., Garboczi E.J., Microstructure and transport properties of porous building materials II. Three dimensional X-ray tomography studies, Mat. Struct., 2000;33:147-153.

Biswal B., Manwart C., Hilfer R., Bakke S., Øren P.E., Quantitative analysis of experimental and synthetic microstructures for sedimentary rock, Physica A.,1999;273:452-475.

Bruand A., Prost R., Effect of water content on the fabric of a soil material: an experimental approach, J. Soil Sci., $1987 ; 38: 461-472$.

Burlion N., Bernard D., Chen D., X-ray microtomography: Application to microstructure analysis of a cementitious material during leaching process, Cem. Concr. Res., 2006;36:346-357.

Cnudde V., Jacobs P.J.S., Monitoring of weathering and conservation of building materials through non-destructive X-ray computed microtomography, Environ. Geol., 2004a;46:477-485.

Cnudde V., Cnudde J.P., Dupuis C., Jacobs P.J.S., X-ray micro-CT used for the localization of water repellents and consolidants inside natural building stones, Mater. Charact., 2004b;53:259-271.

Coles M.E., Hazlett R.D., Spanne P., Soll W.E., Muegge E.L., Jones K.W., Pore level imaging of fluid transport using synchrotron X-ray microtomography, J. Pet. Sci. Eng.,1998;19:55-63.

Cousin I., Levitz P., Bruand A., Three-dimensional analysis of a loamyclay soil using pore and solid chord distributions, Eur. J. Soil Sci., 1996;47:439-452.

D'Acqui L.P., Bruand A., Pagliai M., Study of soil porosity with mercury porosimetry and image analysis on backscattered electron scanning images (BESI). Application to tilled "crusting soils" in Zimbabwe. Soil micromorphology: studies in management and genesis. Proceedings of the IX International Working Meeting on Soil Micromorphology, Townsville, Australia, July 1992, Dev. Soil Sci. 22, 581-590.

De Graef B., Cnudde V., Dick J., De Belie N., Jacobs P., Verstraete W., A sensitivity study for the visualisation of bacterial weathering of concrete and stone with computerised X-ray microtomography, Sci. Total Environ., 2005;341:173-183

Frykman P., Rogon T.A., Anisotropy in pore networks analyzed with 2-D autocorrelation (variomaps), Comput. Geosci., $1993 ; 19: 887-930$.

Gallucci E., Scrivener K., Groso A., Stampanoni M., Margaritondo G., 3-D experimental investigation of the microstructure of cement pastes using synchrotron X-ray microtomography $(\mu C T)$, Cem. Concr. Res., 2007;37:360-368.

Hoshen J., Kopelman R., Percolation and cluster distribution I. Cluster multiple labelling technique and critical concentration algorithm, Phys. Rev. B, 1976;14:3438-3445.

Ioannidis M.A., Kwiecien M.J., Chatzis I., Statistical analysis of the porous microstructure as a method for estimating reservoir permeability, J. Pet. Sci. Eng., 1996;16:251-261. 
Ketcham R.A., Carlson W.D., Acquisition, optimization and interpretation of X-ray computed tomographic imagery: applications to the geosciences, Comput. Geosci., 2001;27:381-400.

Le Trong E., Rozenbaum O., Rouet J.L., Bruand A., A simple methodology to segment X-ray tomographic images of a multiphasic building stone, Image Anal. Stereol., 2008;27:175-182.

Levitz P., Tchoubar D., Disordered porous solids: from chord distribution to small angle scattering, J. Phys I., 1992;2:771790.

Lindquist W.B., Venkatarangan A., Investigating 3-D geometry of porous media from high resolution images, Phys. Chem. Earth A,, 1999;24:593-599.

Lindquist W.B., Quantitative analysis of three-dimensional x-ray tomographic images, Developments in X-Ray Tomography III, in: Ulrich Bonse (Eds.), SPIE Proceedings Series, 4503, 2002:103-115.

Maire E., Colombo P., Adrien J., Babout L., Biasetto L., Characterization of the morphology of cellular ceramics by 3D image processing of X-ray tomography, J. Eur. Ceram. Soc, 2007, 27, 1973-1981.

Parra Denis E., Barat C., Jeulin D., Ducottet C., 3D complex shape characterization by statistical analysis: Application to aluminium alloys, Mat. Char., 2008, 59, 338-343.

Pierret A., Capowiez Y., Belzunces L., Moran C.J., 3-D reconstruction and quantification of macropores using X-ray computed tomography and image analysis, Geoderma, 2002;106:247-271.

S. Rolland du Roscoat, M. Decain, X. Thibault, C. Geindreau, J.-F. Bloch, Estimation of microstructural properties from synchrotron X-ray microtomography and determination of the REV in paper materials , Acta Mater., 2007, 55, 2841-2850.

Rozenbaum O., Le Trong E., Rouet J.L., Bruand A., 2-D image analysis: A complementary tool for characterizing quarry and weathered building limestone, J. Cult. Heritage, 2007;8:151-159.

Spanne P., Thovert J.F., Jacquin C.J., Lindquist W.B., Jones K.W., Adler P.M., Synchrotron computed microtomography of porous media: topology and transports, Phys. Rev. Let., 1994;73:2001-2004.

Stampanoni M., Borchert G., Wyss P., Abela R., Patterson B., Hunt S., Vermeulen D., Ruegsegger P., High resolution X-ray detector for synchrotron-based microtomography, Nucl. Inst.Meth. A, 2002;491:291-301.

Tafforeau P., Bentaleb I., Jaeger J.J., Martin C., Nature of laminations and mineralization in rhinoceros enamel using histology and X-ray synchrotron microtomography: Potential implications for palaeoenvironmental isotopic studies, Palaeogeogr Palaeocl 246 (2007) 206-227

Török Á., Rozgonyi N, Morphology and mineralogy of weathering crusts on highly porous oolitic limestone, a case study from Budapest, Environ. Geol. 2004;46:333-349.

Vignoles G.L., Image segmentation for phase-contrast hard X-ray CMT of C/C composites, Carbon, 2001;39:167-173.

Vogel H.J., Roth K., Quantitative morphology and network representation of soil pore structure, Adv. Water Res., $2001 ; 24: 233-242$.

Vogel H.J., 2008. QuantIm - Some useful C/C++ functions for scientific image processing. Helmholtz Center for Environmental Research, Halle, http://www.ufz.de/index.php?en=16562. 


\begin{tabular}{|c|c|c|c|c|c|c|c|c|c|}
\hline Sample & \multicolumn{2}{|c|}{ Grey level } & \multicolumn{2}{|c|}{ Volume fraction } & \multicolumn{2}{c|}{ Volume of percolating cluster } & $\chi_{\mathrm{V}}\left(\mu \mathrm{m}^{-3}\right)$ \\
\hline & 11 & lh & pore & silica & calcite & pore & silica & calcite & \\
\hline W0-1 & 109 & 164 & 0.467 & 0.347 & 0.185 & $249.10^{6}$ & $185.10^{6}$ & $37.10^{6}$ & $-32.10^{-6}$ \\
\hline W1-2 & 109 & 152 & 0.289 & 0.415 & 0.295 & $152.10^{6}$ & $222.10^{6}$ & $146.10^{6}$ & $-12.10^{-6}$ \\
\hline W2-3 & 113 & 161 & 0.296 & 0.418 & 0.286 & $156.10^{6}$ & $223.10^{6}$ & $145.10^{6}$ & $-22.10^{-6}$ \\
\hline UW & 113 & 158 & 0.228 & 0.481 & 0.290 & $117.10^{6}$ & $258.10^{6}$ & $146.10^{6}$ & $-19.10^{-6}$ \\
\hline
\end{tabular}




\begin{tabular}{|c|c|c|c|c|c|c|c|c|}
\hline Sample & $\begin{array}{c}\text { 2 phase chord } \\
\text { momentum }\end{array}$ & \multicolumn{4}{|c|}{ Correlation length $(\mu \mathrm{m})$} & \multicolumn{4}{|c|}{ Anisotropy } \\
\hline & $\mathrm{l}_{\mathrm{s}} / 1_{\mathrm{p}}$ & $\mathrm{L}$ & $\mathrm{L}_{\mathrm{x}}$ & $\mathrm{L}_{\mathrm{y}}$ & $\mathrm{L}_{\mathrm{z}}$ & $\mathrm{x}$ & $\mathrm{y}$ & $\mathrm{z}$ \\
\hline W0-1 & 1.1 & 18.8 & 25.3 & 27.9 & 11.1 & -0.6 & -0.8 & 0.3 \\
\hline W1-2 & 2.2 & 21.6 & 26.5 & 13.9 & 23.7 & -0.2 & 0.3 & -0.1 \\
\hline W2-3 & 2.1 & 18.5 & 22.1 & 13.0 & 20.4 & -0.2 & 0.3 & -0.1 \\
\hline UW & 3.0 & 13.0 & 15.6 & 14.2 & 12.0 & -0.2 & -0.1 & 0.1 \\
\hline
\end{tabular}


Weathered surface

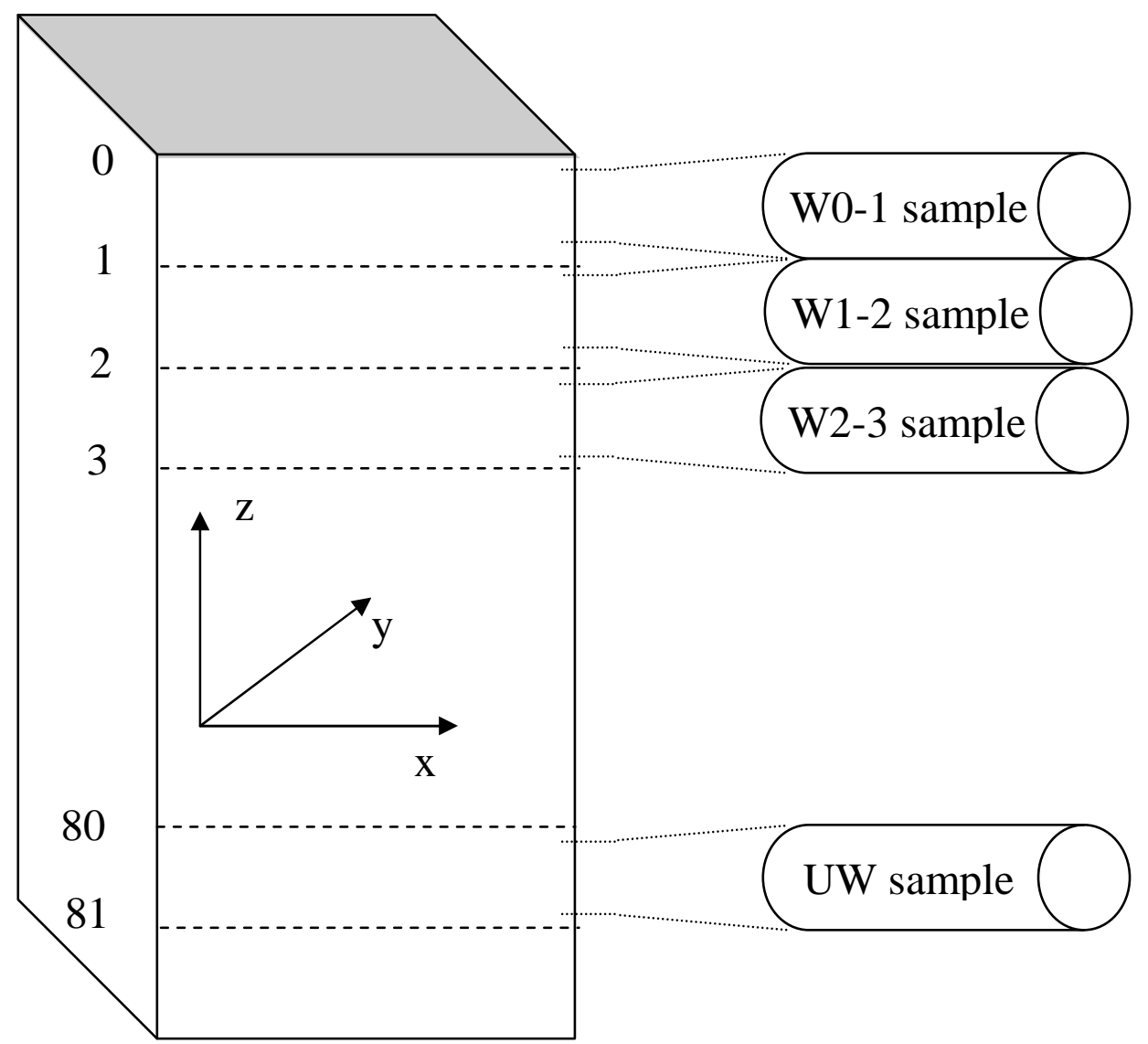

Unweathered core 

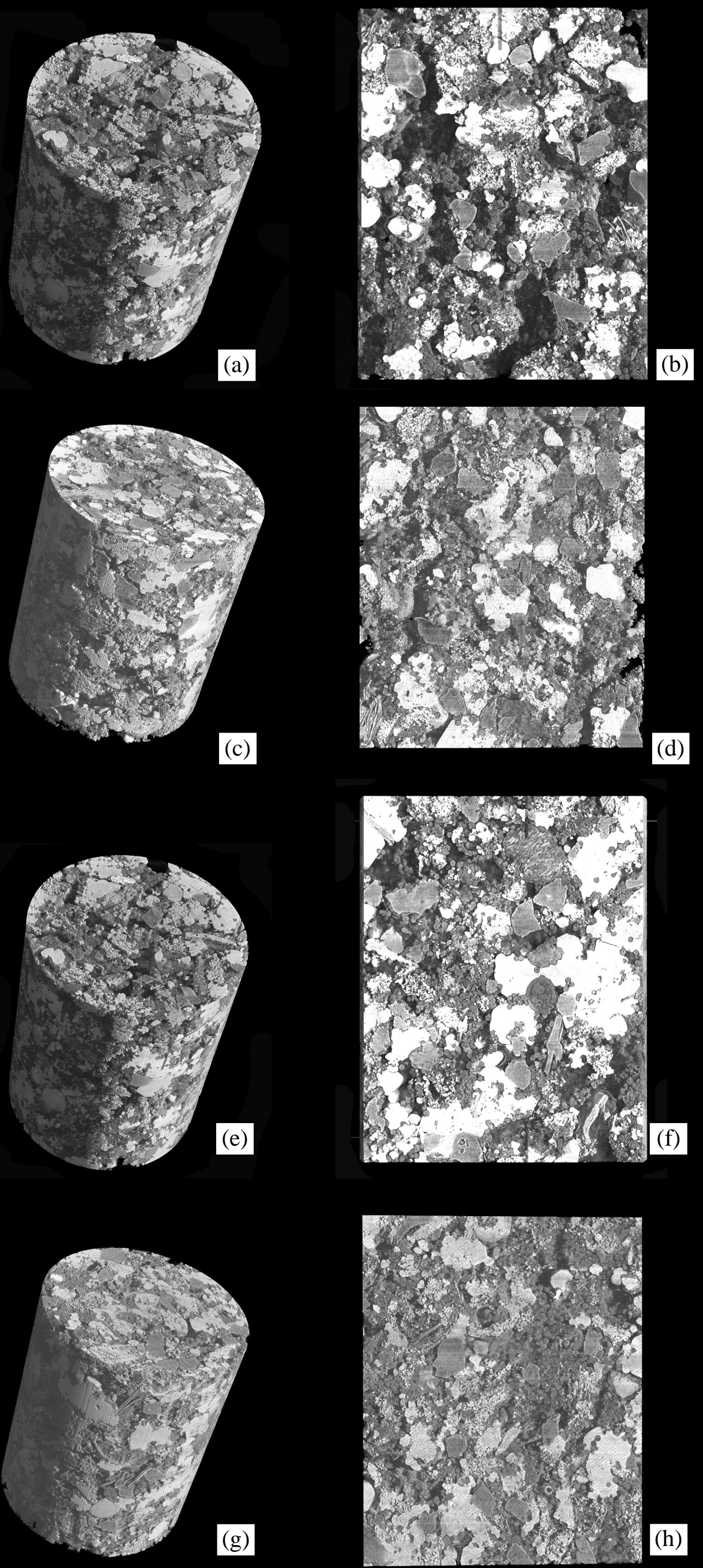


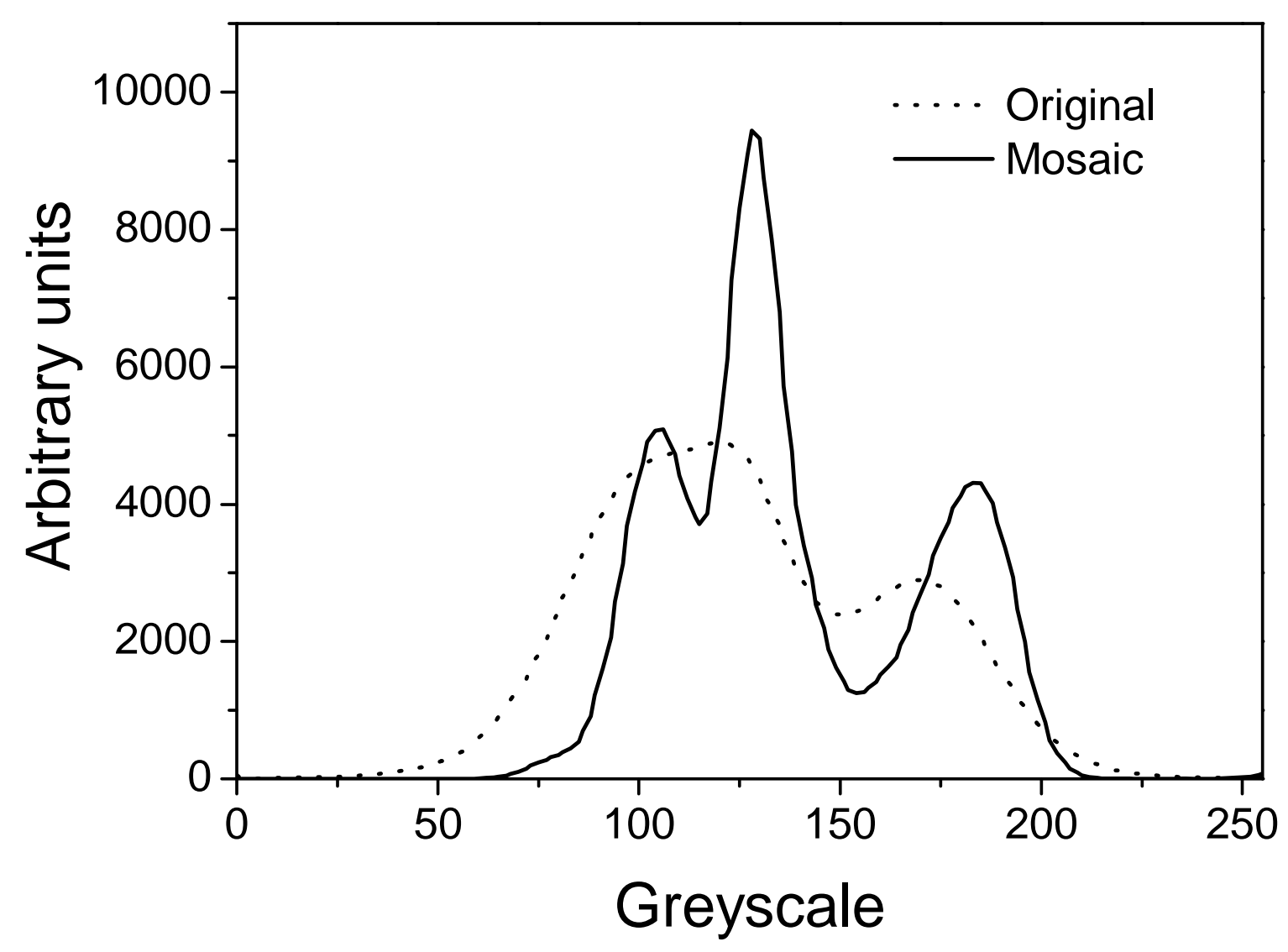



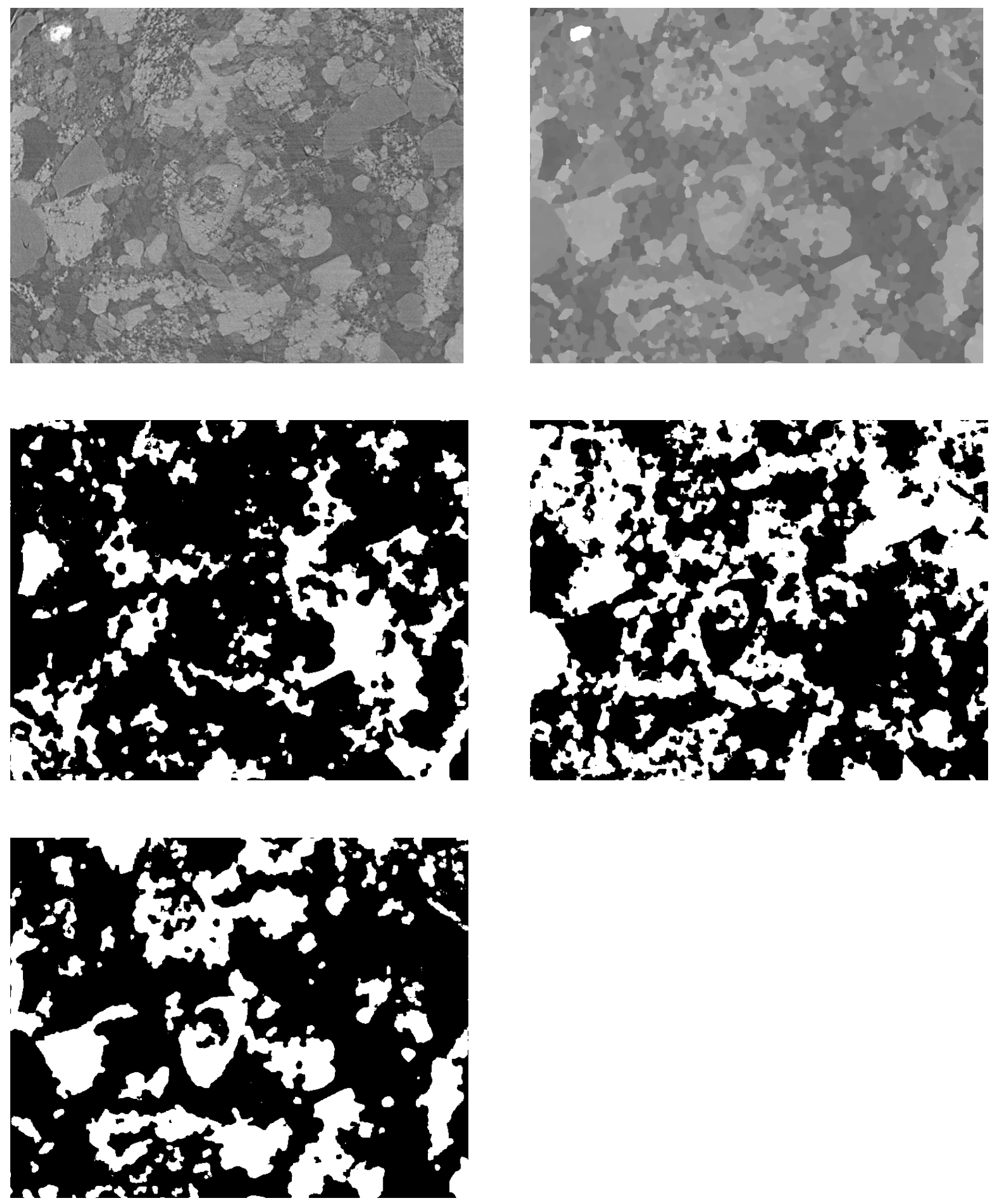

Original image, mosaic

Pore silice calcite 

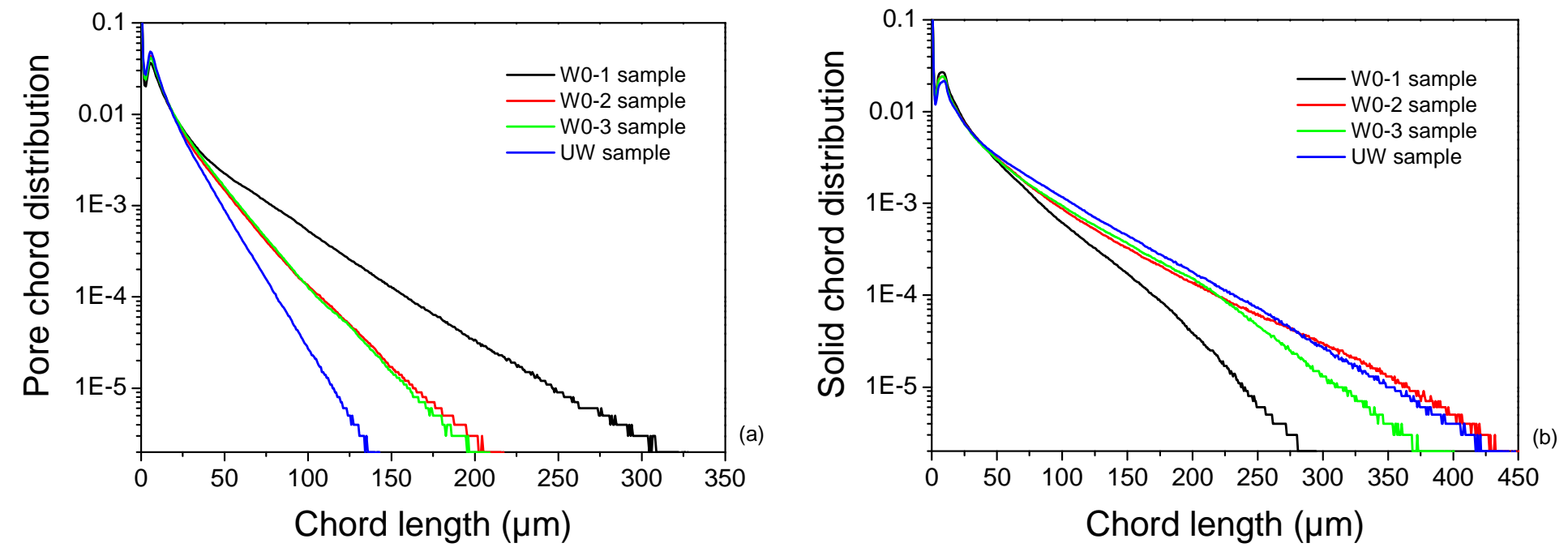

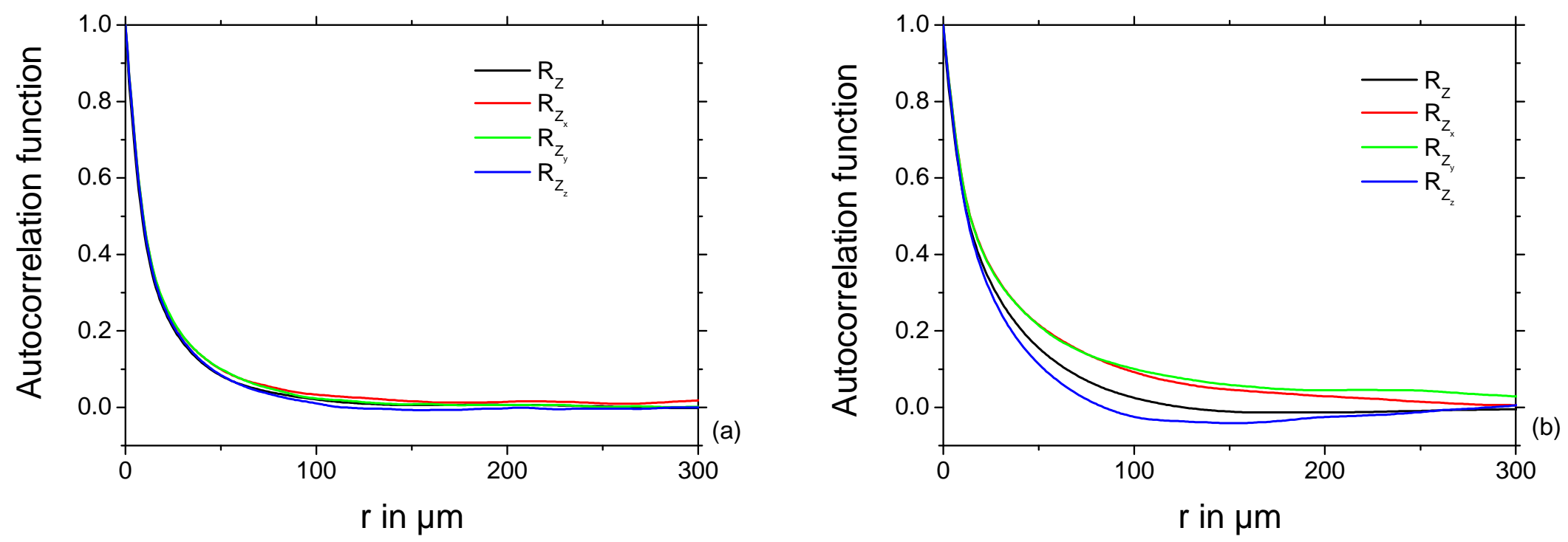